\title{
Impairment of Selectin-mediated Leukocyte Adhesion to Venular Endothelium in Spontaneously Hypertensive Rats
}

\author{
Makoto Suematsu, ${ }^{\ddagger}$ Hidekazu Suzuki, Takuya Tamatani, ${ }^{\S}$ Yutaka ligou, ${ }^{\ddagger}$ Frank A. DeLano, Masayuki Miyasaka," \\ Michael J. Forrest, “ Reiji Kannagi,' Benjamin W. Zweifach, Yuzuru Ishimura, ${ }^{\ddagger}$ and Geert W. Schmid-Schönbein \\ Department of Bioengineering and Institute for Biomedical Engineering, University of California, San Diego, La Jolla, California \\ 92093-0412; *Cytel Co., La Jolla, California 92121; ${ }^{\ddagger}$ Department of Biochemistry, School of Medicine, Keio University, Tokyo 160, \\ Japan; ${ }^{\S}$ Pharmaceutical Basic Research Laboratories, Japan Tobacco Inc., Yokohama 236, Japan; "Department of Bioregulation, \\ Biomedical Research Center, Osaka University Medical School, 565 Osaka, Japan; and "Laboratory of Experimental Pathology, \\ Aichi Cancer Center, Nagoya 464, Japan
}

\begin{abstract}
The present study was designed to elucidate whether molecular mechanisms for leukocyte adhesion to microvascular endothelium may differ between spontaneously hypertensive rats and Wistar Kyoto rats. Leukocyte rolling and adhesion were investigated while monitoring venular wall shear rates in the mesenteric microcirculation stimulated with histamine or tert-butyl hydroperoxide in the two strains. In Wistar Kyoto rats, $10 \mu \mathrm{M}$ histamine as well as $500 \mu \mathrm{M}$ tertbutyl hydroperoxide promoted a significant reduction of venular leukocyte rolling velocity and subsequent adhesion. These changes in leukocyte behavior were blocked by monoclonal antibodies against P-selectin (PB 1.3) and against sialyl Lewis X-like carbohydrates (2H5). However, spontaneously hypertensive rats exhibited a blunted response of the stimulus-elicited leukocyte rolling, which was associated with impairment of venular P-selectin expression as well as a decrease in the expression of sialyl Lewis X-like carbohydrates on circulating neutrophils. No significant differences were detected between the two strains not only in the surface CD11b/CD18 expression but also in the CD18-mediated adhesivity of neutrophils to intracellular adhesion molecule-1 transfectants in vitro. These results suggest that impairment of selectin-mediated leukocyte adhesion is an event responsible for disorders of inflammatory responses in spontaneously hypertensive rats. (J. Clin. Invest. 1995. 96:20092016.) Key words: leukocyte adhesion - endothelial cells • P-selectin • sialyl Lewis X • hypertension
\end{abstract}

\section{Introduction}

The progressive elevation of arterial blood pressure in humans as well as in various experimental models of hypertension, is associated with an array of microcirculatory derangements that develop early in the formative stages of this pathological condi-

Address correspondence to Geert W. Schmid-Schöbein, Department of Bioengineering and Institute for Biomedical Engineering, University of California San Diego, 9500 Gilman Drive, La Jolla, CA 92093-0412. Phone:619-534-3852; FAX:619-534-5722.

Received for publication 14 January 1994 and accepted in revised form 26 June 1995.

J. Clin. Invest.

(C) The American Society for Clinical Investigation, Inc.

0021-9738/95/10/2009/08 \$2.00

Volume 96, October 1995, 2009-2016 tion $(1,2)$. Despite the extensive literature on hypertensive syndromes, there is as yet no consensus with respect to the factors which predispose hypertensive subjects to specific organ complications. We have reported a significant increase in the number of spontaneously activated neutrophils and monocytes in the pool of circulating leukocytes in spontaneously hypertensive as well as Dahl hypertensive rats $(3,4)$. Such high numbers of circulating phagocytes may contribute to the pathogenesis of organ complications in the hypertensives. According to recent reports $(5,6)$, however, spontaneously hypertensive rats (SHR ${ }^{1}$ show a suppressed leukocyte adhesion to endothelium in response to various proinflammatory reagents compared with Wistar Kyoto (WKY) rats. But the molecular mechanism for such an attenuation of leukocyte-endothelial interaction remains unresolved.

The major objective of the present study was to compare the ability of venular endothelial cells to elicit leukocyte adhesion in SHR and normotensive WKY and to investigate the molecular mechanisms responsible for dysfunction of the leukocyte adherence in hypertensives. In vivo video microscopic measurements in the mesenteric microcirculation have revealed that the stimulus-dependent venular leukocyte rolling is significantly blunted, suggesting that the disorder of selectin-mediated adhesion mechanisms plays a fundamental role in impairment of leukocyte-endothelial interactions in SHR.

\section{Methods}

Animal preparation. Male SHR and age-matched normotensive WKY were obtained from Charles River Laboratories (Wilmington, MA). Animals were housed in a controlled environment and maintained on a standard pellet diet for at least $7 \mathrm{~d}$ before initiation of the experimental procedures. 12-13-wk-old SHR and WKY (280-320 grams) were anesthetized with sodium pentobarbital ( $40 \mathrm{mg} / \mathrm{kg}$, IM), $30 \mathrm{~min}$ prior to exposure of the ileocecal mesentery for intravital microvascular observation. The femoral artery and vein were cannulated with PE-50 polyethylene tubes (Clay Adams, Parsippany, $\mathrm{NJ}$ ) to monitor systemic arterial pressure and to inject reagents, respectively. The rats were placed on a heating pad, covered with a blanket, and maintained at $37^{\circ} \mathrm{C}$. The abdomen was opened via a midline incision. The ileocecal portion of the mesentery was carefully exposed and mounted on a plastic pedestal for intravital microscopy as described previously $(7-10)$. The preparation was kept at $37^{\circ} \mathrm{C}$ and continuously superfused with Krebs-Henseleit bicarbonate-buffered solution ( $\mathrm{pH} 7.4$ ) saturated with a $95 \% \mathrm{~N}_{2} / 5 \%$ $\mathrm{CO}_{2}$ gas mixture.

1. Abbreviations used in this paper: CFSE, carboxyfluorescein diacetate succinimidyl ester; CHO, Chinese hamster ovary; SHR, spontaneously hypertensive rat; t-BH, tert-butyl hydroperoxide; WKY, Wistar Kyoto rat. 
Intravital microscopy. The mesenteric microcirculation was visualized through an intravital microscope $(\times 55$ water immersion objective E Leitz Wetzlar GmbH, Wetzlar, Germany ) via a CCD television camera (model 2362; Javelin Electronics, Tokyo, Japan) to investigate venular leukocyte behavior while monitoring arteriolar and venular erythrocyte velocity (11). Single unbranched venules ( $25-40 \mu \mathrm{m}$ in diameter) were selected for study. Centerline erythrocyte velocity $\left(V_{\mathrm{R}}\right)$ was measured by the cross-correlation velocimeter (IPM, Inc., La Mesa, CA) and was calibrated against a rotating glass disk coated with erythrocytes rotating with known velocity. Arteriolar and venular blood flow was computed from the product of mean blood cell velocity $\left(V_{\text {mean }}=\right.$ centerline velocity/1.6) (12) and microvascular cross-sectional area, assuming a cylindrical lumen crossection. Venular diameter $\left(D_{v}\right)$ was measured using the video image-shearing technique (IPM, Inc.). Venular wall shear rate $(\gamma)$ was calculated based on the definition for a Newtonian fluid: $\gamma=$ $8\left(V_{\text {mean }} / D_{\mathrm{v}}\right)(13)$

The number of adherent leukocytes was determined off-line during play back of the videotape images. A leukocyte was regarded to be adherent to venular endothelium when it remained stationary for at least $30 \mathrm{~s}$. Adherent cells were expressed as the number per $100-\mu \mathrm{m}$ length of venular segment. The velocity of rolling leukocytes was determined by counting the number of individual video frames required for a leukocyte to pass over a preselected distance along the venular wall. In addition, to evaluate details of the leukocyte-endothelial cell interaction, the distance over which a leukocyte was rolling as well as the time to traverse the same distance was measured, and the mean leukocyte rolling velocity for individual leukocytes was then computed. To compose a histogram for the normalized leukocyte velocity $\left(V_{\mathrm{W}} / V_{\mathrm{R}}\right)$, such measurements were repeated for at least 50 rolling cells in each experiment. $V_{\mathrm{W}} / V_{\mathrm{R}}$ is an index of the membrane adhesive stress between leukocytes and endothelium (14). Approximately 3-min video records were usually required to complete 50 measurements.

In vivo endothelium-leukocyte interactions after stimulation. After the mesentery was exposed and prepared on a temperature-controlled stage, the preparation was stabilized for $\sim 20 \mathrm{~min}$. During this period, all hemodynamic parameters measured on-line (arterial pressure, erythrocyte velocity, arteriolar, and venular diameter) reached a steady state, and venular leukocyte rolling mediated by P-selectin expression was almost abolished ( see Results). To investigate the stimulated endothelial cell-dependent mechanisms for leukocyte rolling and adhesion in the hypertensives and their controls, histamine dihydrochloride (10 $\mu \mathrm{M}$; Sigma Chemical Co., St. Louis, MO) or tert-butyl hydroperoxide ([ $\mathrm{t}$ $\mathrm{BH}$ ] $500 \mu \mathrm{M}$; Sigma Chemical Co.) was continuously superfused on the mesenteric preparation of WKYs and SHRs. The mAbs used in the study were PB1.3 (anti-P-selectin; Cytel Corp., La Jolla, CA) (15), 2H5 (anti-sialyl LeX-like carbohydrates, mouse IgM) (16) (17), and HRL-3 and HRL-4 (anti-L-selectin) (14). HRL-3 and HRL-4 were $\mathrm{F}\left(\mathrm{ab}^{\prime}\right)_{2}$ fragments of anti-L-selectin mAbs. Previous experiments have shown that pretreatment with HRL-3, but not with HRL-4, increases venular leukocyte rolling velocity in rats (18). As a control, immunoglobulin for PB1.3, mouse $\operatorname{IgG}_{1}$ (Sigma Chemical Co.) was used in another set of experiments. Each $\mathrm{mAb}$ or mouse $\mathrm{IgG}_{1}$ was administered intravenously by way of the femoral vein at a dose of $2.0 \mathrm{mg} / \mathrm{kg}, 15 \mathrm{~min}$ before the application of proinflammatory reagents, such as histamine or t-BH (Sigma Chemical Co.). None of the mAbs used in this study caused a significant change in the number of circulating leukocytes or neutrophils in WKY $(18,19)$ or in SHR (data not shown).

In vivo endothelium-leukocyte interaction in the low-flow state. In another set of experiments, the effects of lowering the venular shear rate on leukocyte-endothelial cell interactions were evaluated in WKY and SHR. In this protocol, two cotton threads were inserted around the local segment of the mesenteric artery proximal to the region under observation. One string was firmly fixed to the animal stage, and another was stretched to reduce the local venular shear rates to values $<250$ / $\mathrm{s}$ for $30 \mathrm{~min}$. The adherent leukocyte density and the local venular shear rate were correlated with the objective to identify differences between WKY and SHR. The density of venular leukocyte adhesion was estimated in two to three different venular segments in one experiment at
20 min after the start of the low-shear condition $(<250 / \mathrm{s})$. In these experiments, a leukocyte which remained at the same position on the venular endothelium for more than $30 \mathrm{~s}$ was regarded as an adherent cell, and the adherent leukocyte density was expressed as the number per unit venular length $(100 \mu \mathrm{m})$

Measurements of erythrocyte velocity, inner vascular diameter, leukocyte rolling velocity, and adhesion density were collected before and every $10 \mathrm{~min}$ after the start of histamine or t-BH superfusion, or after the start of the low-shear period. To evaluate differences in the distribution of the relative leukocyte velocity $\left(V_{\mathrm{W}} / V_{\mathrm{R}}\right)$ between WKY and SHR, histograms were prepared with $>500$ measurements derived from six to eight animals in each group.

In vivo monitoring of platelet-endothelial cell interactions. To explore whether the effects of the anti-P-selectin mAb (PB 1.3) on leukocyte adhesion might be attributable to the up-regulation of P-selectin on activated platelets, in vivo platelet-endothelial cell interaction was monitored microscopically during the histamine superfusion using a novel fluorochrome, carboxyfluorescein diacetate succinimidyl ester (CFSE; Molecular Probes, Eugene, OR) $(20,21) .1 .0 \mathrm{mg} / \mathrm{kg}$ of the CFSE solution (dissolved in DMSO) was diluted with $1.5 \mathrm{ml}$ physiological saline, and injected at $0.3 \mathrm{ml} / \mathrm{min}$ into the femoral vein, $30 \mathrm{~min}$ before the histamine superfusion. CFSE-labeled platelets were visualized through a fluorescence microscope $(\times 55$ water-immersion objective lens; E. Leitz Wetzlar $\mathrm{GmbH}$ ) equipped with a silicon intensified target camera (C-2400-08; Hamamatsu Photonics, Hamamatsu, Japan). CFSE-labeled platelets were identified as pin-point fluorescent activities flowing along the microvessels (20). The exposure time for recording CFSE microfluorographs was limited to a maximum of $40 \mathrm{~s}$ during each measurement to minimize photobleaching. Platelets which remained at the same position on the venular endothelium for at least $30 \mathrm{~s}$ were regarded as stationary platelets. The number of such platelets per 100 $\mu \mathrm{m}$ venular segment length was estimated at 10 -min intervals after the application of histamine.

Immunohistochemistry of $P$-selectin in the rat mesenteric microcirculation. To elucidate the endothelial P-selectin expression in WKY and SHR, immunohistochemical analysis was carried out according to a previously modified method $(22,23)$. Briefly, $30 \mathrm{~min}$ after the histamine superfusion, the blood supply to the intestine was ligated close to the abdominal aorta and then cut (24). Vascular tissue samples were taken after intravenous infusion of the anti-P-selectin mAb PB1.3 $(2 \mathrm{mg} / \mathrm{kg})$ $50 \mathrm{~min}$ before fixation. The circulating mouse $\mathrm{mAb}$ could attach only to luminal P-selectin and was subsequently detected with an anti-mouse IgG antibody as described elsewhere $(22,23)$. The mesenteric tissues were immediately immersed in absolute methanol $\left(-20^{\circ} \mathrm{C}\right)$ and allowed to stand for $12 \mathrm{~h}$ at $-20^{\circ} \mathrm{C}$. The mesentery was permeablized in $0.5 \%$ Triton X-100 (Sigma Chemical Co.,) for $10 \mathrm{~min}$, and was incubated with $3 \%$ hydrogen peroxide for 10 min to block the endogenous peroxidase activity. After rinsing in PBS, the mesentery was exteriorized on the glass slide and incubated for $2 \mathrm{~h}$ with $3 \%$ bovine serum albumin to block nonspecific binding to the tissue. At the end of the incubation, biotinylated anti-mouse IgG (Dako Co., Carpinteria, CA) was used as a secondary antibody. The incubation was carried out at room temperature for $60 \mathrm{~min}$ with the appropriate dilution by streptavidin peroxidase (Dako Co., Carpinteria, CA) in $0.02 \mathrm{M}$ PBS ( $\mathrm{pH} 7.0$ ) containing $0.02 \%$ hydrogen peroxide for $5 \mathrm{~min}$ in a dark chamber. The tissues were counterstained with hematoxylin before mounting. Concurrent controls included mesenteric tissues where the primary antibody (PB 1.3) was substituted with physiological saline or nonbinding isotype-matched mouse IgG.

Detection of L-selectin, sialyl LeX-like carbohydrates, and integrins in circulating leukocytes. To explore differences in the expression of adhesion molecules on neutrophils, flow cytometric analysis was carried out using FITC-labeled mAbs directed against CD11b (WT-5), CD18 (WT-3), L-selectin (HRL4), and sialyl LeX (2H5). WKY and SHR were anesthesized and blood samples were drawn into EDTA-containing cuvettes by heart puncture, as described previously (18). Constitutive expression of these adhesion molecules on neutrophils was determined using an EPICS Elite flow cytometer (Coulter Electronics, Hialeah, FL) 
and the FITC-labeled monoclonal antibodies. 10,000 cells were analyzed in each experiment which was carried out on blood samples from five pairs of WKYs and age-matched SHRs. The peak fluorescence intensity of the histogram in each pair of measurements (arbitrary units) was estimated and the measurement derived from SHR was expressed as a percentage versus that from the paired WKY. The effects of $50 \mathrm{ng} / \mathrm{ml}$ PMA on the molecular expression were also tested using the same system.

Evaluation of neutrophil adhesion to rat intracellular adhesion molecule-1 (ICAM-1)-transfected Chinese hamster ovary ( $\mathrm{CHO})$ cells. To evaluate the difference between WKY and SHR in the CD11b/CD18dependent adhesion of neutrophils, rat ICAM-1-transfected Chinese hamster ovary cells (CHO-R28) and their negative control cells (CHOK1) were cultured according to a method described elsewhere (25). Neutrophils in the marginating pool were isolated from WKY and SHR by a hetastarch exchange transfusion (26). The cell suspension $\left(1 \times 10^{6}\right.$ cell $/ \mathrm{ml}$ ) was incubated with $10 \mu \mathrm{M} 2^{\prime}, 7^{\prime}$-bis (carboxyethyl)-5(6) carboxyfluorescein tetra-acetoxymethyl ester (BCECF-AM; Dojindo Laboratories, Kumamoto, Japan) for $1 \mathrm{~h}$ at $37^{\circ} \mathrm{C}$, washed and resuspended in the modified Eagle's medium (MEM), and served as a sample for cell binding assay (27). Unstimulated neutrophils $\left(1 \times 10^{6}\right.$ cells/ $\mathrm{ml})$ or neutrophils stimulated with phorbol myristate acetate $(50 \mu \mathrm{g} /$ $\mathrm{ml}$, for $10 \mathrm{~min}$ ) were inoculated onto the wells containing $\mathrm{CHO}$ cells in a final volume of $100 \mu \mathrm{l}$ and incubated for $30 \mathrm{~min}$ at $37^{\circ} \mathrm{C}$. The well plates were then inverted for $15 \mathrm{~min}$ at room temperature to remove nonadherent neutrophils. After the adherent cells were lysed with $50 \mu \mathrm{l}$ of $0.1 \%$ NP- 40 , the number of these cells was calculated on the basis of a fluorescence readout at $530 \mathrm{~nm}$ under the excitation at $490 \mathrm{~nm}$ using an automated microplate fluorometer (MTP; Corona Electric Co., Ibaraki, Japan) (27). Effects of pretreatment with mAbs against CD18 (WT-3, $10 \mu \mathrm{g} / \mathrm{ml}$ at final concentration) or against ICAM-1 (1A29, $\mathrm{F}\left(\mathrm{ab}^{\prime}\right)_{2}$ fragments, $16 \mu \mathrm{g} / \mathrm{ml}$ at final concentration) were evaluated in selected experiments.

Statistics. Significance of the difference in these values between groups was determined by analysis of variance and Scheffe-type multiple comparison test. Differences in the mean and SD values in the histogram of the leukocyte rolling velocity among groups were statistically evaluated by a nonparametric Wilcoxon's ranking test and Kolmogorov-Smirnov test, respectively, according to previous methods (18). Mann-Whitney's U-test was used to compare the peak fluorescence intensity between the two strains in flow cytometry studies. All values are expressed as mean $\pm \mathrm{SD}$, and statistical significance was set at a value of $P<0.05$.

\section{Results}

Spontaneous leukocyte rolling in WKY and SHR. The mean blood pressure measured in the femoral artery at the beginning of the experiments ( $30 \mathrm{~min}$ after anesthesia) was $118 \pm 10 \mathrm{vs}$ $160 \pm 18 \mathrm{mmHg}(P<0.05, n=8)$ in WKY and SHR, respectively. As previously reported, the circulating leukocyte counts $(5,260 \pm 1,280$ vs $8,700 \pm 3,870$ cells $/ \mu 1, n=8)$ and neutrophil counts $(1,120 \pm 630$ vs $2,030 \pm 1,060$ cells $/ \mu \mathrm{l}, n=8)$ were significantly higher in SHR than in WKY $(P<0.05)$ at the age used for these experiments. Fig. 1 illustrates leukocyte rolling velocity normalized by the erythrocyte velocity $\left(V_{\mathrm{W}} / V_{\mathrm{R}}\right)$ in the unstimulated venules of WKY and SHR. During the control period before administration of $\mathrm{mAbs}, V_{\mathrm{W}} / V_{\mathrm{R}}$ histograms could be closely approximated by a log-normal distribution with a median value of $\sim 2-3 \%$ of the centerline erythrocyte velocity in both species. No difference in distribution and median values of the histograms were observed in the two strains. Pretreatment with PB1.3 did not alter the distribution of the normalized leukocyte rolling velocity in the resting period before the histamine application both in WKY and in SHR. In contrast, the anti-
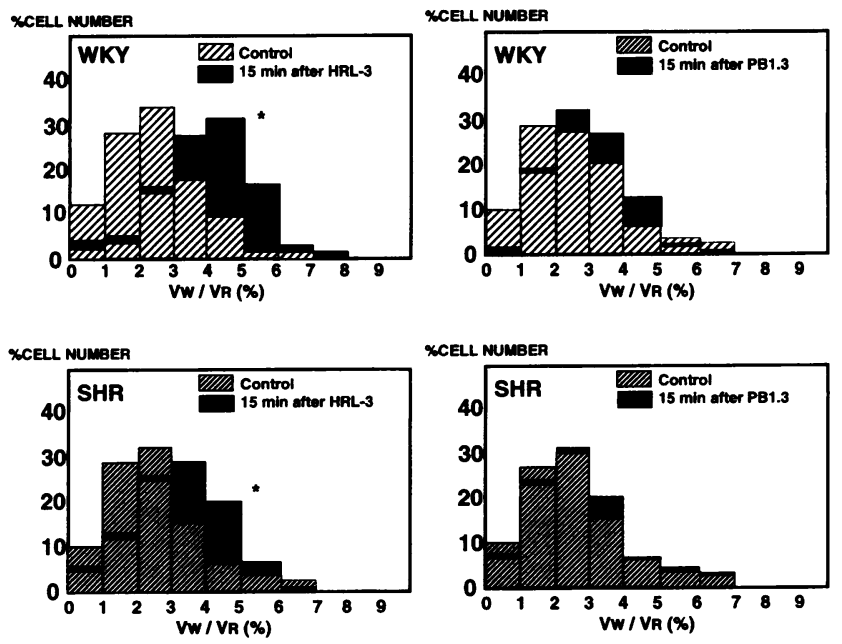

Figure 1. Histogram of the leukocyte rolling velocity in unstimulated mesenteric venules of normotensive Wistar Kyoto $(W K Y)$ and spontaneously hypertensive rats $(S H R)$, and the effects of monoclonal antibodies against rat $\mathrm{L}-(H R L-3)$ and $\mathrm{P}$-selectins $(P B 1.3)$. The value $\mathrm{V}_{\mathrm{w}} / \mathrm{V}_{\mathrm{R}}(\%)$ denotes the leukocyte rolling velocity $\left(V_{W}\right)$ normalized by the centerline erythrocyte velocity $\left(V_{R}\right)$. Each histogram was prepared from 500 measurements of the rolling velocity in six to eight animals for each group. Note that treatment with HRL-3 but not with PB1.3 induces a significant increase in the normalized rolling velocity in the two strains, suggesting the involvement of L-selectin in spontaneous leukocyte rolling. $* P<$ 0.05 , indicating statistically significant difference in the histogram distribution.

L-selectin $F\left(a b^{\prime}\right)_{2}$ mAb (HRL-3) significantly increased the relative leukocyte rolling velocity both in WKY and in SHR. These results indicate that in both species the molecular interaction with L-selectin contributes to spontaneous leukocyte rolling in venules in the unstimulated venules.

Histamine-induced leukocyte activation in WKY and SHR. Fig. 2 illustrates the time history of erythrocyte velocity, wall shear rates, and leukocyte rolling velocity in venules of WKY and SHR during histamine treatment. After superfusion of 10 $\mu \mathrm{M}$ histamine, WKY showed a significant decrease in the leukocyte rolling velocity concomitant with a transient increase in flow volume and venular shear rate. In contrast, in SHR, the same concentration of histamine neither induced any significant decrease in the leukocyte rolling velocity nor evoked a transient increase in venular shear rates.

To explore the possible contribution of P-selectin to the histamine-induced leukocyte-endothelial cell interaction, the relative leukocyte velocity $\left(V_{\mathrm{W}} / V_{\mathrm{R}}\right)$, the wall shear rate and the number of adherent cells in venules were compared among the two groups (Fig. 3). After histamine superfusion for $30 \mathrm{~min}$ in WKY, the relative leukocyte rolling velocity was significantly decreased, suggesting an increasing adhesive stress between venular endothelium and leukocyte membranes. Pretreatment with $\mathrm{PB} 1.3$ or with $2 \mathrm{H} 5$ significantly blunted these changes, suggesting the involvement of P-selectin and SLeX in the histamine-induced increase in adhesive force. As pointed out previously $(28,29)$, pretreatment with a histamine $\mathrm{H}_{1}$ receptor antagonist (diphenhydramine) did suppress the histamine-elicited responses, implicating the involvement of $\mathrm{H}_{1}$ receptormediated mechanism (data not shown). SHR, however, exhibited no significant changes in leukocyte rolling in response to 

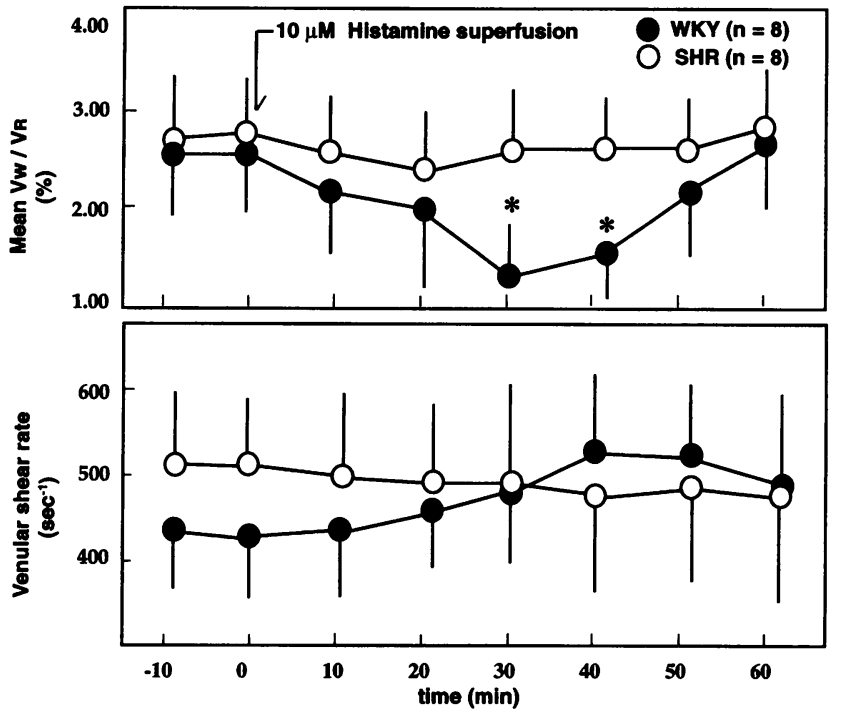

Figure 2. Effects of histamine superfusion $(10 \mu \mathrm{M})$ on venular shear rates and the normalized leukocyte rolling velocity in WKY and SHR. The mean ratio $V_{\mathrm{W}} / V_{\mathrm{R}}$ was computed as an average of individual median values in each of eight experiments for two strains. To determine the median value in each rat, $\sim 50$ measurements of the rolling velocity were carried out in each experiment. Histamine induces a significant transient reduction of the normalized leukocyte rolling velocity in WKY, but not in SHR. $* P<0.05$ as compared with the value in SHR.

histamine, and pretreatment with $\mathrm{PB} 1.3$ or $2 \mathrm{H} 5$ did not alter these parameters, suggesting an impairment of histamine-induced leukocyte rolling mechanisms. Although histamine elicited a slight increase in the number density of adhesion (not significant), the magnitude was still $<50 \%$ of that in WKY.

Hydroperoxide-induced leukocyte rolling in WKY and SHR. To test the hypothesis that the difference in P-selectin-mediated leukocyte rolling between WKY and SHR is not just the result of a difference in histamine sensitivity between the two strains, the effect of $\mathrm{t}-\mathrm{BH}$, another stimulator for P-selectin expression (22) was evaluated. In WKY, $500 \mu \mathrm{M}$ t-BH superfusion induced a significant reduction of $V_{\mathrm{W}} / V_{\mathrm{R}}$ without significant decrease in the venular shear rate and with a time history distinct from that induced by histamine (Fig. 4). Pretreatment with PB1.3 or $2 \mathrm{H} 5$, but not with mouse IgG or IgM, attenuated these changes, suggesting again the involvement of P-selectin and SLeX in the $\mathrm{t}-\mathrm{BH}$-elicited adhesion reaction in WKY rats. The t-BH-elicited reponses of leukocyte rolling seemed to be independent of endogenous histamine release, inasmuch as neither diphenhydramine nor ranitidine, an inhibitor of histamine $\mathrm{H}_{2}$ receptor, attenuated the reduction of leukocyte rolling velocity (data not shown). The adhesive response after t-BH application were likewise blunted in SHR (Fig. 5). These results suggest that the down-regulation of the selectin-mediated leukocyte rolling in SHR occurs independently of the choice of stimulus.

Platelet-endothelial cell interactions elicited by histamine and $t-B H$ in vivo. To investigate whether the preventive effects of an $\mathrm{P}$-selectin $\mathrm{mAb}$ during histamine- or $\mathrm{t}-\mathrm{BH}$-elicited leukocyte rolling and adhesion in WKY is due to immunoneutralization of platelet P-selectin, in vivo platelet-endothelial cell interactions were visually investigated using CFSE-assisted digital microfluorography. Table I illustrates the density of platelet adhering to the venular endothelium exposed to histamine or $\mathrm{t}$ -
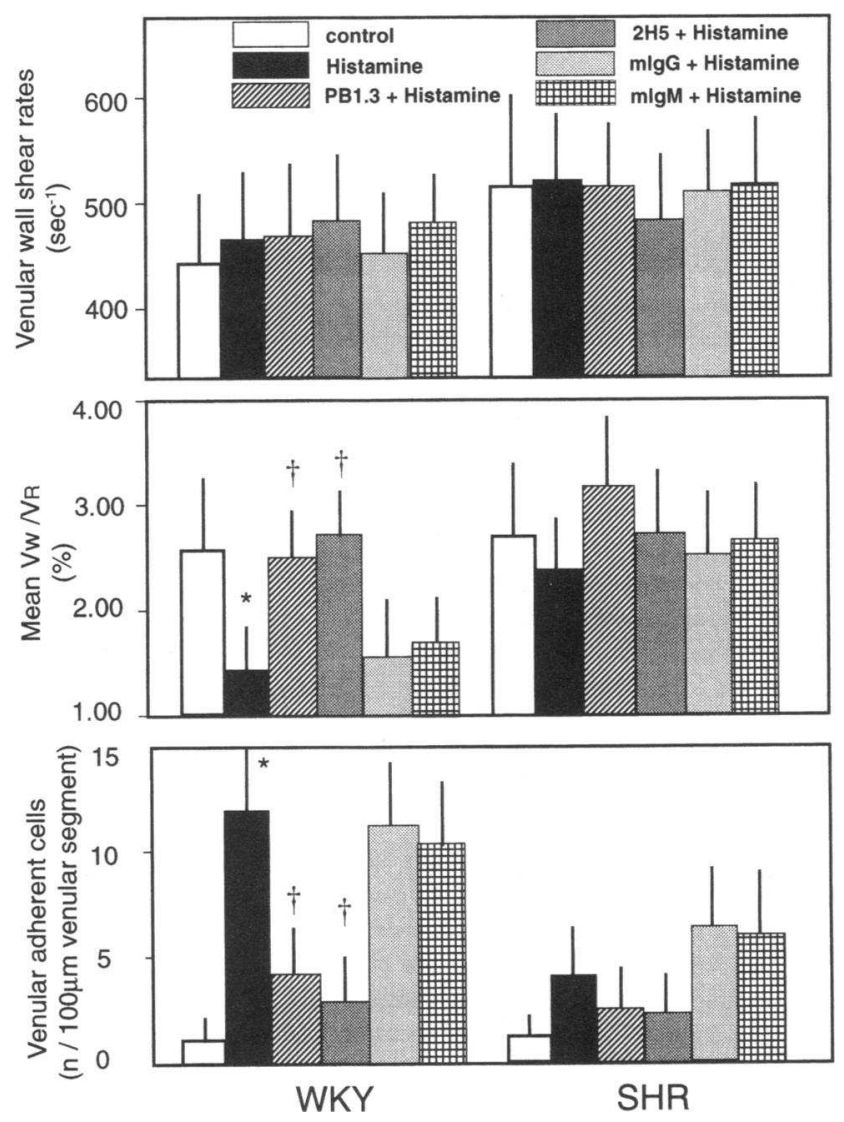

Figure 3. Effects of pretreatment with a monoclonal antibody against P-selectin ( $P B 1.3, n=6$ in the same strain) and against SLeX (2H5, $n=5$ in the same strain) on histamine-induced leukocyte-endothelial cell interactions in venules of WKY and SHR. Measurements were carried out $30 \mathrm{~min}$ after initiation of histamine superfusion. Histamineinduced reduction of the leukocyte rolling velocity and enhancement of the venular adhesion were significantly suppressed by a P-selectindirected monoclonal antibody PB1.3. 2H5 also decreased the histamineinduced leukocyte activation. $\mathrm{mIgG}$ and $\mathrm{mIgM}$ are mouse IgG and IgM as negative controls ( $n=5$ in the same strain) for the PB1.3- and 2H5treated groups, respectively. The number of animals in the histaminetreated groups in each strain is eight. $* P<0.05$ as compared with the control values. In SHR, the histamine-induced reduction of the rolling velocity and adherent changes were significantly blunted. ${ }^{\dagger} P<0.05$ as compared with the histamine-treated WKY group.

$\mathrm{BH}$ for $30 \mathrm{~min}$. In the two strains, neither histamine nor $\mathrm{t}$ BH did elicit a significant increase in the number of platelet aggregates adhering to the venules. It is therefore conceivable that the attenuating effect of PB1.3 on histamine- or t-BHinduced activation of leukocyte rolling and sticking is attributable to its blockade of endothelial P-selectin rather than on activated platelets.

Differences in microvascular expression of P-selectin in $W K Y$ and SHR. To elucidate the mechanisms for the impairment of histamine-promoted leukocyte rolling in SHRs, differences in microvascular P-selectin expression were determined by immunohistochemistry in the mesentery of WKY and SHR (Fig. 6 ). While the histamine-treated mesenteric microvessels in WKY exhibited a positive immunostaining (Fig. $6 b$ ), microvessels in SHR showed only a negligible level of staining (Fig. $6 d$ ), indicating that histamine-elicited P-selectin expression in endothelial cells may be blunted in the hypertensives. 

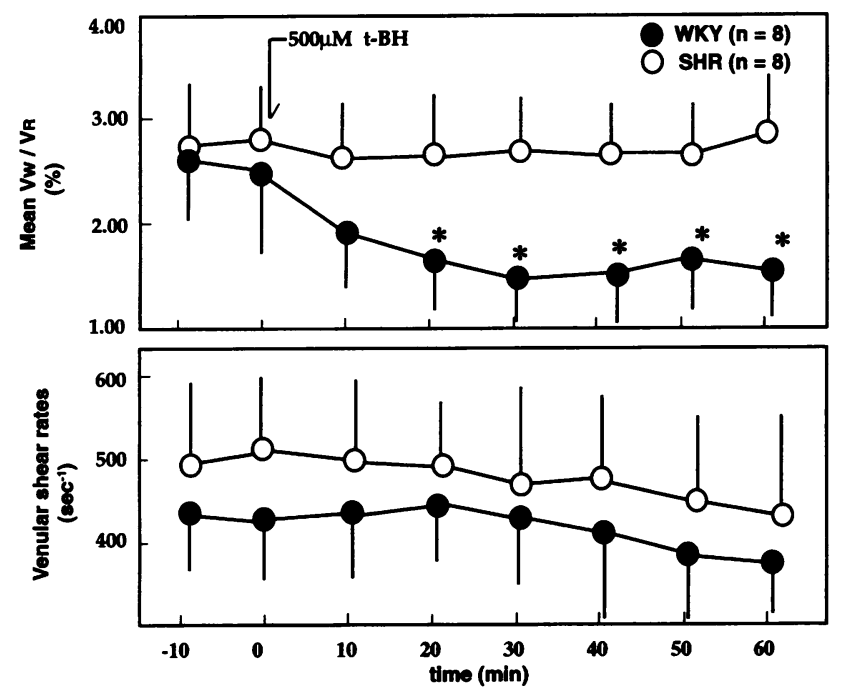

Figure 4. Time course in the leukocyte rolling velocity and shear rates in venules during tert-butyl hydroperoxide $(t-B H, 500 \mu M)$ superfusion in WKY and SHR. The mean $V_{\mathrm{W}} / V_{\mathrm{R}}$ was estimated from eight experiments in the two strains as described in the legend of Fig. 2. Note that $\mathrm{t}-\mathrm{BH}$ induces a persistent reduction of the normalized leukocyte rolling velocity in WKY, but not in SHR. ${ }^{*} P<0.05$ as compared with the value in SHR.

Down-regulation of SLeX-like carbohydrates on neutrophils in SHR. To clarify further the difference in adhesive reactions of circulating neutrophils to endothelium between WKY and SHR, the expression of L-selectin, SLeX, and two subunits of integrins (CD11b, CD18) were determined by laser flow cytometry and fluorescence-labeled monoclonal antibodies (Fig. 7). In the resting stage, before addition of phorbol myristate acetate as a stimulus, the expression of CD11b and CD18 was not significantly different between neutrophils of WKY and those of SHR. The neutrophil L-selectin was expressed in SHR at a slightly lower level than in WKY. The most striking difference between the two strains was observed in the expression

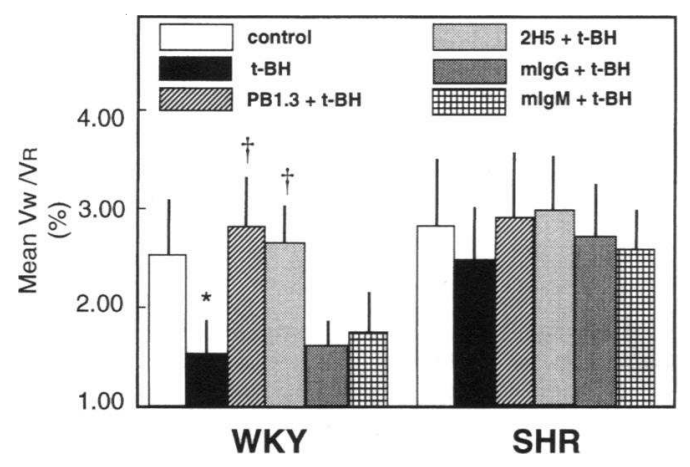

Figure 5. The normalized leukocyte rolling velocity after treatment with tert-butyl hydroperoxide $(t-B H, 500 \mu \mathrm{M})$. The effects of pretreatment with monoclonal antibodies against $\mathrm{P}$-selectin $(P B 1.3)$ and against SLeX-like carbohydrates (2H5). $\mathrm{mIgG}$ and $\mathrm{mIgM}$ are mouse IgG and IgM as negative controls ( $n=5$ in the same strain) for the PB1.3- and $2 \mathrm{H} 5$-treated groups, respectively. The number of rats in other groups is eight. $* P<0.05$ as compared with the control values. In SHRs, the t-BH-elicited response is significantly blunted. ${ }^{\dagger} P<0.05$ as compared with the t-BH-treated WKY group.
Table I. The Density of CFSE-labeled Platelets Adhering to the Venular Endothelium during the Superfusion with Histamine or $t-B H$ in WKY and SHR

\begin{tabular}{llc}
\hline & \multicolumn{2}{c}{$\begin{array}{c}\text { The density of adherent platelets } \\
(n / 100-\mu \mathrm{m} \text { venular segment })\end{array}$} \\
\cline { 2 - 3 } & Control & 30 min after the stimulus \\
\hline WKY & & \\
Histamine $(10 \mu \mathrm{M}, n=5)$ & $2.4 \pm 1.0$ & $2.5 \pm 1.2$ \\
t-BH $(500 \mu \mathrm{M}, n=5)$ & $2.6 \pm 1.2$ & $3.2 \pm 1.6$ \\
SHR & & \\
Histamine $(10 \mu \mathrm{M}, n=5)$ & $1.8 \pm 1.3$ & $1.9 \pm 1.6$ \\
t-BH $(500 \mu \mathrm{M}, n=5)$ & $2.0 \pm 1.4$ & $2.3 \pm 1.4$ \\
\hline
\end{tabular}

Data represent the number of adherent platelets before (Control), and $30 \mathrm{~min}$ after the superfusion of stimuli. Neither histamine nor t-BH elicits a significant increase in the density.

of SLeX-like carbohydrates: the peak intensity of the 2H5associated fluorescence in neutrophils of SHR ranged at as low as $42.8 \pm 10.3 \%(P<0.01)$ of that of WKY. Although the expression of these molecules was measured in lymphocytes derived from the two species, there were no significant differences between WKYs and SHRs (data not shown). After stimulation with PMA, neutrophils in the both species exhibited down-regulation of L-selectin and SLeX with simultaneous upregulation of integrins. These results suggest that down-regulation of SLeX-like carbohydrates in circulating leukocytes may serve as another possible mechanism for impairment of P-selectin-dependent leukocyte recruitment in SHR.

Preservation of low shear-dependent leukocyte adhesion in SHR. If alteration in the selectin-mediated adhesion mechanism is a critical event which results in the impaired leukocyte rolling in the histamine- or t-BH-stimulated venules of SHR, the difference in the leukocyte-endothelium interaction between the two strains might be diminished under low shear conditions, since the low shear-dependent leukocyte adhesion is thought to be mediated by the mechanism dependent on ICAM- 1 and CD11/CD18 rather than by that dependent on selectins (19). We therefore explored the effects of reduced shear rates on leukocyte-endothelial cell interaction in the venules of WKY and SHR (Fig. 8). $30 \mathrm{~min}$ after the start of the low shear condition, the density of venular-adherent leukocytes significantly increased in WKY as well as in SHR. These results suggest that the difference in the leukocyte rolling behavior between the two strains can be observed only under physiological shear rates.

Leukocyte adhesion of SHR neutrophils to ICAM-1-expressed $\mathrm{CHO}$ cells. Since there is no significant difference between WKY and SHR in the amount of CD11b/CD18 expression, we have attempted to examine another possibility that the functional difference in this integrin may be attributable to that of histamine- or t-BH-induced neutrophil adhesion between the two strains. We tested in vitro neutrophil binding of the rat ICAM-1-transfected CHO-R28 cell as well as of its negative control CHO-K1 cell to neutrophils derived from WKYs and SHRs (Fig. 9). Adherence of unstimulated neutrophils from WKYs to the ICAM-1 - transfected CHO-R28 cells was $\sim$ fourfold greater than that to CHO-K1 cells. Furthermore, coincubation with WT-3 as well as with $\mathrm{F}\left(\mathrm{ab}^{\prime}\right)_{2}$ fragments of $1 \mathrm{~A} 29$ 


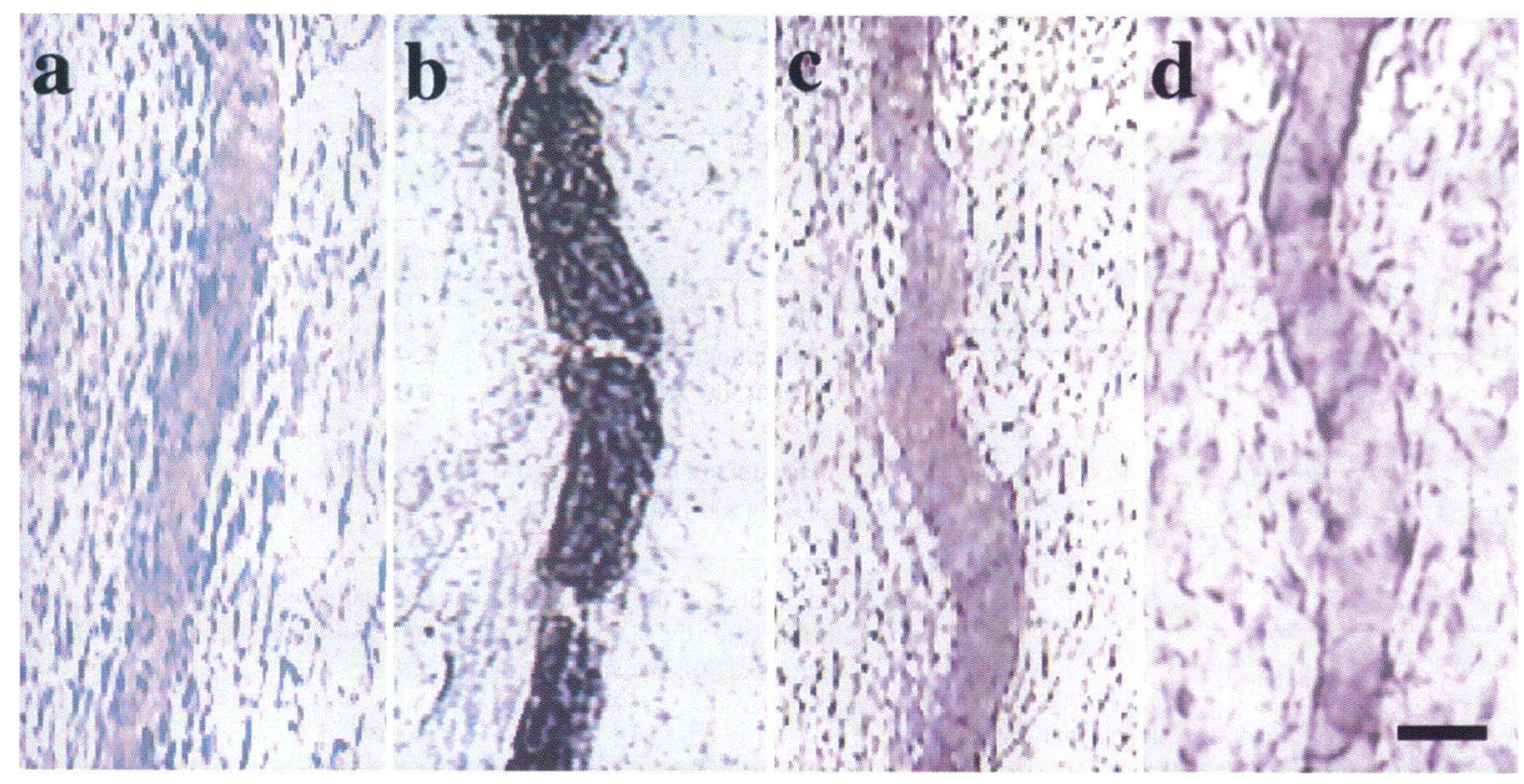

Figure 6. P-selectin expression determined by immunohistochemistry in the rat mesentery. Photomicrographs of the postcapillary venules in whole mount show the immunohistochemical staining after treatment with mAb against P-selectin (PB1.3). (a) The mesenteric venule in WKY rats without treatment of histamine. There is no brown staining in the field. (b) 30 min after treatment with histamine, strong brown staining (positive staining) in the intravascular lumen was shown in WKY rats. (c) Mesenteric venule in SHR without treatment of histamine. No detectable levels of brown staining were present in the venule of SHR mesentery. $(d)$ The mesenteric venule in SHR 30 min after treatment of histamine. No detectable levels of positive staining were present. Bar indicates $50 \mu \mathrm{m}$.

attenuated the neutrophil binding to CHO-R28 cells almost completely, indicating that the cell adherence in this system results from the specific binding between ICAM-1 and CD18. Unstimulated neutrophils from SHRs exhibited a similar extent of adherence to $\mathrm{CHO}-\mathrm{R} 28$ cells, illustrating no significant difference in the magnitude of adhesion between the two strains. In addition, treatment with WT-3 or with $F\left(a^{\prime}\right)_{2}$ fragments of 1 A29 abolished the adherence of neutrophils from SHR in the same manner as was observed when those from WKY were applied, suggesting that the CD18-dependent neutrophil adhesivity results from specific binding to ICAM-1 on CHO-R28. Adherence of neutrophils stimulated with PMA did not exhibit any significant difference between the two strains (data not shown). These results suggest that there is no fundamental difference in CD18-dependent adhesivity of circulating neutrophils between the two strains.

\section{Discussion}

The results of the present study demonstrate that SHR exhibits impairment of leukocyte rolling and adhesion in postcapillary venules treated with histamine, which is known to elicit significant leukocyte rolling in normotensive rats $(6,23,28,29)$. These findings provide several possible explanations as to why
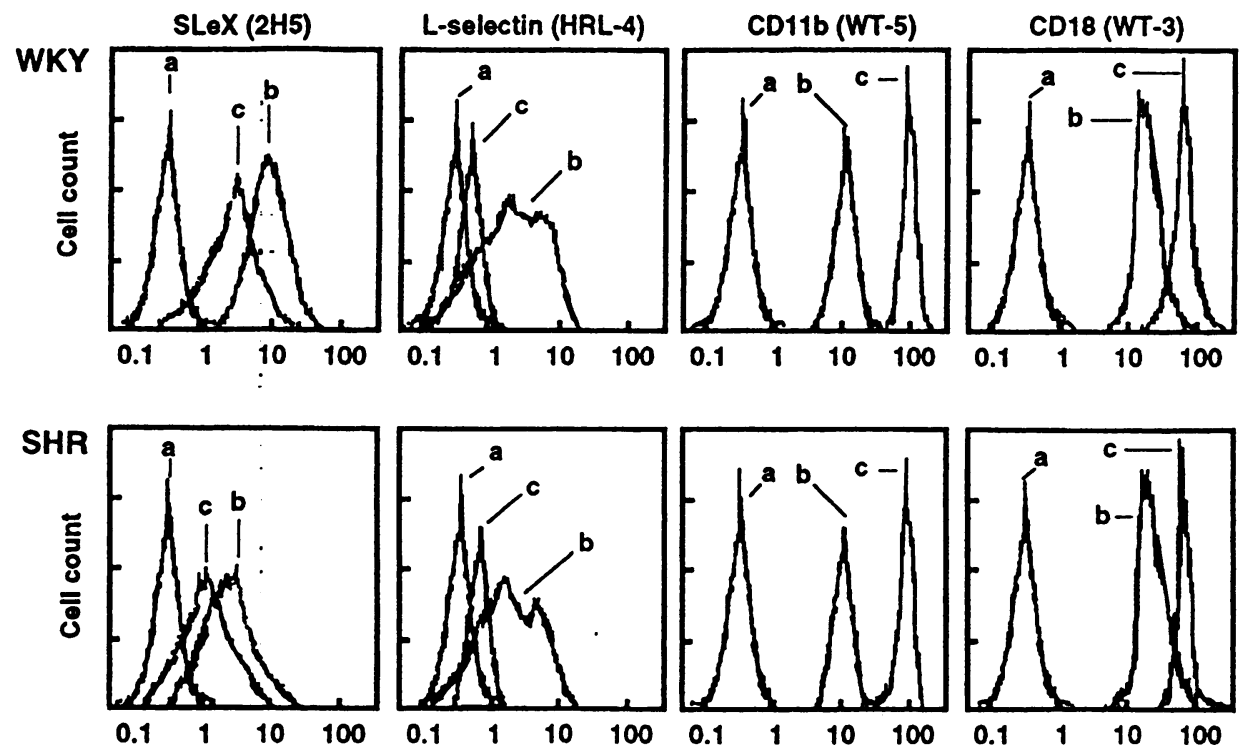

Figure 7. Flow cytometric histograms of the expression of sialyl Lewis X, Lselectin, $\mathrm{CD} 11 \mathrm{~b}$, and $\mathrm{CD} 18$ on circulating neutrophils derived from WKY (top) and SHR (bottom). (a) Background fluorescence levels. 2H5, HRL4, WT-5, and WT-3 are monoclonal antibodies directed against SLeX, L-selectin, CD11b, and CD18, respectively. (b) Histogram before stimulation with $50 \mathrm{ng} / \mathrm{ml}$ PMA. (c) Histogram after stimulation with $50 \mathrm{ng} / \mathrm{ml}$ PMA. 


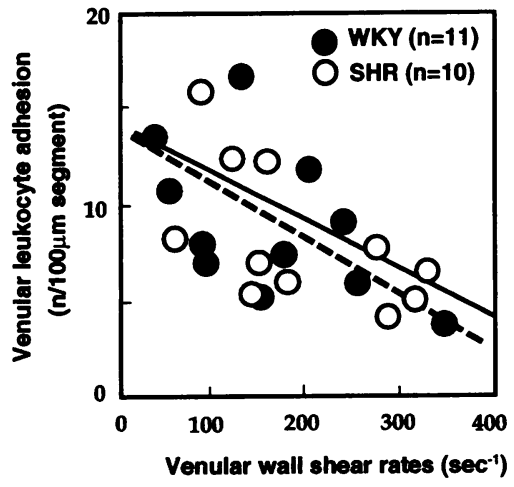

Figure 8 . The number of leukocytes adhering to postcapillary venules in WKY and SHR during reduction of shear rate. The period for the wall shear reduction was 30 min in all experiments. The density of adherent leukocytes was expressed as the number of adherent cells per a 100 $\mu \mathrm{m}$ local venular segment. The regression line between venular wall shear rate $(x)$ and venular leukocyte adhesion $(y)$ is $\mathrm{y}=-0.025 \mathrm{x}+13.7$ (solid line: $n=10, r=0.66$ ) in WKY and $\mathrm{y}$ $=-0.027 \mathrm{x}+13.8$ (broken line: $n=11, r=0.82$ ) in SHR.

histamine-induced leukocyte rolling is blunted in SHR. First, the histamine receptor responsible for the leukocyte rolling could be down-regulated in the mesentery. However, this possibility seems unlikely, since another stimulator such as t-BH which promotes receptor-independent P-selectin expression (30) can also activate leukocyte rolling in WKY, and the response is likewise blunted in SHR. Secondly, the intracellular signal transduction pathway leading to P-selectin expression where histamine shares roles with t-BH in endothelial cells (e.g. mechanisms for translocation of Weibel-Palade bodies) may differ between the two strains (30). Recent observations from our and other laboratories suggest that endothelial cell-dependent arteriolar relaxation via nitric oxide is severely impaired in SHR because of overproduction of superoxide anions in vascular endothelial cells (31-34). Although this possibility was not examined in the present study, circumstances such as longterm exposure to oxidative stress in SHR might alter normal expression mechanisms for adhesion molecules in endothelial cells involving receptor translocation and reinternalization. Interestingly, it has recently been reported that tissue ICAM-1 contents in the splanchnic organs is significantly down-regulated in SHR (35), which may serve as a possible explanation for reduction of neutrophil adhesion elicited by FMLP or platelet activating factor (5). In addition, the current study provides evidence for novel mechanisms responsible for the blunted leukocyte rolling and adhesion in SHR: down-regulation of luminal $\mathrm{P}$-selectin expression in venules and a decrease in the surface expression of SLeX-like carbohydrates on circulating neutrophils.

Impairment of the endothelial selectin pathway in SHR provides further insight into possible mechanisms for some of the disorders of leukocyte function in this strain that have been reported previously. First, the increase in the number of circulating neutrophils and monocytes ( 3 ) may result from demargination of these cells because of chronic suppression of selectindependent cell margination (6). This hypothesis has recently been buttressed by results from P-selectin gene knockout mice which show a significant increase in the circulating neutrophil counts (36). Secondly, SHR may exhibit a diminished sensitivity to inflammatory stimuli relative to WKY because of the impairment of P-selectin-mediated pathway for leukocyte recruitment. Recent studies suggest that P-selectin mediates a rolling interaction between neutrophils and endothelial cells which in turn facilitates neutrophil-directed juxtacrine activa-

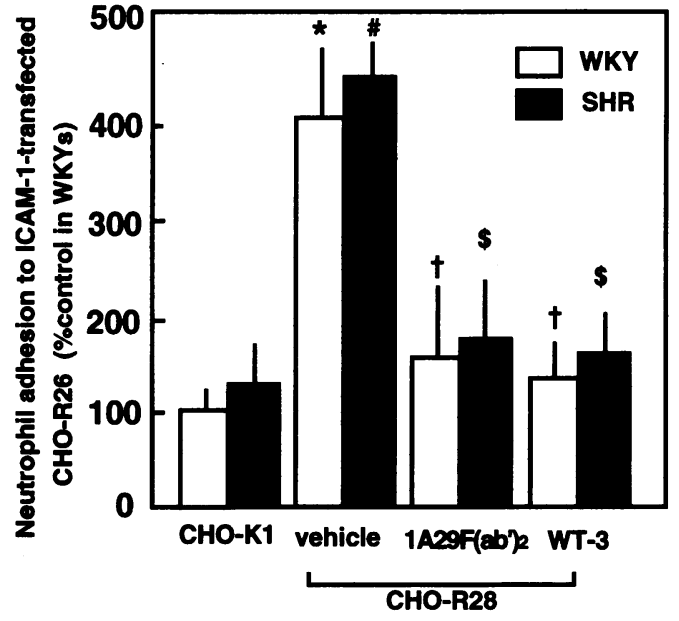

Figure 9. Adherence of neutrophils from WKY and SHR to ICAM-1 transfected $\mathrm{CHO}$ cell $(\mathrm{CHO}-\mathrm{R} 28)$ and to its mock transfectant $(\mathrm{CHO}$ $K 1)$ in vitro. The adhesion density was expressed as percentage values versus that in the control (CHO-K1-neutrophil binding) of WKY. Open and thick bars represent the values (mean \pm SD of four experiments) obtained from neutrophils of WKY and SHR, respectively. ${ }^{*, *} P<0.05$ as compared with the respective control values. ${ }^{+. S} P<0.05$ significant inhibition by a monoclonal antibody against CD18 (WT -3$)$ or against ICAM-1 $\left(1 A 29 F\left(a b^{\prime}\right) 2\right)$. There is no significant difference in the neutrophil adhesion to ICAM-1-expressed CHO cells between the two strains.

tion via other signaling molecules in the endothelium, such as platelet activating factor (37). It may therefore be postulated that the impact of neutrophil-mediated injury under physiological shear rates may be considerably weakened in SHR. An analogous hypothesis is supported by several experimental models of gastrointestinal damage $(38,39)$, in which neutrophilmediated injury mechanisms appear to be a factor in determining the severity of lesions (40).

While a P-selectin-dependent leukocyte recruitment appears to be required under normal shear rates, it seems to play a reduced role under low shear rates (21). Interestingly, SHR is known to be more vulnerable to hemorrhagic hypotension than WKY (40). According to our previous observation (41), the population of neutrophils producing superoxide spontaneously in the circulation represents a key factor which determines the sensitivity to hemorrhagic shock.

It is therefore likely that the SHR, which has significantly greater number of spontaneously activated neutrophils in the circulation than WKY (3), is more susceptible to hemorrhagic hypotension (40). In addition, according to Arndt et al. (5), the tissue myeloperoxidase activity in the lung, the major compartment of marginating neutrophils (26), is significantly higher in SHR than in WKY, suggesting an increase in the baseline population of marginating and/or residual neutrophils in and around pulmonary microvessels. Once exposed to hemorrhagic shock, the spontaneously activated neutrophils in the circulation are likely to be trapped within pulmonary microvessels of SHR subjecting the lung to even higher oxidative stress by these activated cells via a selectin independent mechanism. It should further be examined in SHR whether redistribution of circulating neutrophils may jeopardize the viability of particular organs such as lung under hemorrhagic conditions via low shear-depen- 
dent leukocyte recruitment which does not require selectin-mediated adhesion mechanisms.

\section{Acknowledgments}

This work was completed during the tenure of a research fellowship for Dr. Hidekazu Suzuki from the American Heart Association, California Affiliate.

The authors thank Mr. Taro Okitsu in Keio University School of Medicine for his technical support.

This work was supported by U.S. Public Health Service grant HL10881 and in part by HL-43026; by International Research Program of Grant-in-Aid for Scientific Research from the Ministry of Education, Science, and Culture of Japan; and by a grant from Keio University School of Medicine.

\section{References}

1. Schmid-Schönbein, G. W., B. W. Zweifach, F. A. DeLano, and P. Chen 1987. Microvascular tone in a skeletal muscle of spontaneously hypertensive rats. Hypertension (Dallas). 9:164-171.

2. Schmid-Schönbein, G. W., M. Suematsu, and B. W. Zweifach. 1994. The potential role of blood leukocyte interaction in progression of the genetic arterial hypertensive syndrome. In Physiology and Pathphysiology of Leukocyte Adhesion. D. N. Granger and G. W. Schmid-Schönbein, editors. Oxford University Press, New York. 467-484.

3. Schmid-Schönbein, G. W., D. Seiffge, F. A. DeLano, K. Shen, and B. W Zweifach. 1991. Leukocyte counts and activation in spontaneously hypertensive and normotensive rats. Hypertension (Dallas). 17:323-330.

4. Shen, K., F. A. DeLano, B. W. Zweifach, and G. W. Schmid-Schönbein. 1995. Circulating leukocyte counts, activation and degranulation in Dahl hypertensive rats. Circ. Res. 76:276-283.

5. Arndt, H., C. W. Smith, and D. N. Granger. 1993. Leukocyte-endothelial cell adhesion in spontaneously hypertensive and normotensive rats. Hypertension (Dallas). 21:667-673.

6. Suzuki, H., G. W. Schmid-Schönbein, M. Suematsu, F. A. DeLano, M. J. Forrest, M. Miyasaka, and B. W. Zweifach. 1994. Impaired leukocyte-endothelial cell interaction in spontaneously hypertensive rats. Hypertension (Dallas). 24:719-727.

7. Suematsu, M., I. Kurose, H. Asako, S. Miura, and M. Tsuchiya. 1989. In vivo visualization of oxyradical-dependent photoemission during endotheliumgranulocyte interactions in the microvascular bed treated with platelet-activating factor. J. Biochem. 103:355-360.

8. Suematsu, M., G. W. Schmid-Schönbein, R. H. Chavez-Chavez, T. T. Yee, T. Tamatani, M. Miyasaka, F. A. DeLano, and B. W. Zweifach. 1993. In vivo visualization of oxidative changes in microvessels during neutrophil activation. Am. J. Physiol. 264:H881-H891.

9. Suematsu, M., T. Tamatani, F. A. DeLano, M. Miyasaka, M. J. Forrest, H. Suzuki, and G. W. Schmid-Schönbein. 1994. Microvascular oxidative stress preceding leukocyte activation elicited by in vivo nitric oxide suppression. Am. J. Physiol. 266:H2410-H2415.

10. Suzuki, H., M. Suematsu, S. Miura, Y. Y. Liu, K. Watanabe, M. Miyasaka, S. Tsurufuji, and M. Tsuchiya. 1994. Rat CINC/gro: a novel mediator for locomotive and secretagogue activation of neutrophils in vivo. J. Leukocyte Biol. 55:652657.

11. Tompkins, W. R., R. Monti, and M. Intaglietta. 1974. Velocity measurements by self tracking correlator. Rev. Sci. Instrum. 45:647-649.

12. Davis, M. J. 1987. Determination of volumetric flow in capillary tubes using an optical doppler velocimeter. Microvasc. Res. 34:223-230.

13. House, S. D., and H. Lipowsky. 1987. Leukocyte-endothelium adhesion: microdynamics in mesentery of the cat. Microvasc. Res. 34:363-379.

14. Schmid-Schönbein, G. W., R. Skalak, S. I. Simon, and R. L. Engler. 1987. The interaction between leukocytes and endothelium in vivo. Ann. NY Acad. Sci. 516:348-361

15. Mulligan, M. S., M. J. Polley, R. J. Bayer, M. F. Nunn, J. C. Paulson, and P. A. Ward. 1992. Neutrophil-dependent acute lung injury. Requirement for P-selectin (GMP-140). J. Clin. Invest. 90:1600-1607.

16. Sawada, M., A. Takada, I. Ohwaki, N. Takahashi, H. Tateno, J. Sakamoto, and R. Kannagi. 1993. Specific expression of a complex sialyl Lewis X antigen on high endothelial venules of human lymph nodes. Biochem. Biophys. Res. Commun. 193:337-347.

17. Tamatani, T., M. Suematsu, K. Tezuka, N. Hanzawa, T. Tsuji, Y. Ishimura, R. Kannagi, S. Toyoshima, and M. Honma. 1995. Recognition of a consensus carbohydrate structure in the ligands for selectins (CD62s) by a novel antibody against sialyl Lewis $\mathrm{X}$ in vitro and in vivo. Am. J. Physiol. In press.
18. Tamatani, T., F. Kitamura, K. Kuida, M. Shirao, M. Mochizuki, M. Suematsu, G. W. Schmid-Schönbein, K. Watanabe, S. Tsurufuji, and M. Miyasaka. 1993. Characterization of LECAM-1 (L-selectin) by the use of monoclonal antibodies and evidence for the presence of soluble LECAM-1 in rat sera. Eur. J. Immunol. 23:2181-2188.

19. Bienvenu, K., and D. N. Granger. 1993. Molecular determinants of shear rate-dependent leukocyte adhesion in postcapillary venules. Am. J. Physiol. 264:H1504-H1508.

20. Suematsu, M., T. Okitsu, H. Suzuki, G. W. Schmid-Schönbein, and Y. Ishimura. 1994. Intravital observation of platelet-endothelial cell interaction during nitric oxide suppression. In Endothelial Cell Function in Blood Flow. M. Tsuchiya, M. Katori, M. Suematsu, and B. W. Zweifach, editors, Excerpta Medica, Amsterdam. 26-33.

21. Suematsu, M., F. A. DeLano, D. C. Poole, R. L. Engler, M. Miyasaka, B. W. Zweifach, and G. W. Schmid-Schönbein. 1994. Spatial and temporal correlation between leukocyte behavior and cell injury in postischemic rat skeletal muscle microcirculation. Lab. Invest. 70:485-495.

22. Winn, R. K., D. Liggitt, N. B. Vedder, J. C. Paulson, and J. M. Harlan. 1993. Anti-P-selectin monoclonal antibody attenuates reperfusion injury to the rabbit ear. J. Clin. Invest. 92:2042-2047.

23. Suzuki, H., B. W. Zweifach, M. J. Forrest, and G. W. Schmid-Schönbein 1995. Modification of leukocyte adhesion in spontaneously hypertensive rats by adrenal corticosteroids. J. Leukocyte Biol. 57:20-26.

24. Watanabe, K., M. Suematsu, M. Iida, K. Takaishi, Y. Iizuka, H. Suzuki, M. Suzuki, M. Tsuchiya, and S. Tsurufuji. 1992. Effect of rat CINC/gro, a member of the interleukin-8 family, on leukocytes in microcirculation of the rat mesentery. Exp. Mol. Pathol. 56:60-69.

25. Kita, Y., T. Takashi, Y. Iigo, T. Tamatani, M. Miyasaka, and T. Horiuchi. 1992. Sequence and expression of rat ICAM-1. Biochim. Biophys. Acta. 1131:108-110.

26. Williams, J. H., Jr., K. M. Moser, T. Ulich, and M. S. Cairo. 1987. Harvesting the noncirculating pool of polymorphonuclear leukocytes in rats by hetastarch exchange transfusion. J. Leukocyte Biol. 42:455-462.

27. Tamatani, T., M. Kotani, T. Tanaka, and M. Miyasaka. 1991. Molecular mechanisms underlying lymphocyte recirculation. II. Differential regulation of LFA-1 in the interaction between lymphocytes and high endothelial cells. Eur. J. Immunol. 21:855-858.

28. Kubes, P., and S. Kanwar. 1994. Histamine induces leukocyte rolling in postcapillary venules. A P-selectin-mediated event. J. Immunol. 152:3570-3577.

29. Asako, H., I. Kurose, R. Wolf, S. DeFrees, Z. L. Zeng, M. L. Phillips, J. C. Paulson, and D. N. Granger. 1994. Role of $\mathrm{H}_{1}$ receptors and P-selectin in histamine-induced leukocyte rolling and adhesion in postcapillary venules. $J$. Clin. Invest. 93:1508-1515.

30. Patel, K. D., G. A. Zimmerman, S. M. Prescott, R. P. McEver, and T. M. McIntyre. 1991. Oxygen radicals induce human endothelial cells to express GMP140 and bind neutrophils. J. Cell Biol. 112:749-759.

31. Nakazono, K., N. Watanabe, K. Matsumoto, J. Sasaki, T. Sato, and M. Inoue. 1991. Does superoxide underlie the pathogenesis of hypertension? Proc. Natl. Acad. Sci. USA. 88:10045-10048.

32. Suematsu, M., T. Nishino, G. W. Schmid-Schönbein, B. W. Zweifach, and Y. Ishimura. 1994. Cancellation of nitric oxide-mediated microvascular flow regulation by xanthine oxidase-mediated oxidative stress in spontaneously hypertensive rats. Int. J. Microcirc. Clin. Exp. 14(Suppl. 1):224a. (Abstr.)

33. Suzuki, H., A. Swei, B. W. Zweifach, and G. W. Schmid-Schönbein 1995. In vivo evidence for microvascular oxidative stress in spontaneously hypertensive rats. Hydroethidine microfluorography. Hypertension (Dallas). 25:10831089.

34. Suzuki, H., B. W. Zweifach, and G. W. Schmid-Schönbein. 1995. Vasodilator responses of mesenteric arterioles to histamine in spontaneously hypertensive rats. Hypertension (Dallas). In press.

35. Komatsu, M., J. Panes, M. A. Perry, J. M. Russell, and D. N. Granger. 1995. Effects of chronic arterial hypertension on constitutive and induced ICAM1 expression in the splanchnic organs. Gastroenterology. 107:546a. (Abstr.)

36. Mayadas, T. N., R. C. Johnson, H. Rayburn, R. O. Hynes, and D. D. Wagner. 1993. Leukocyte rolling and extravasation are severely compromised in P-selectin-deficient mice. Cell. 74:541-554.

37. Lorant, D. E., M. K. Tophan, R. E. Whatley, R. P. McEver, T. M. McIntyre, S. M. Prescott, and G. A. Zimmerman. 1993. Inflammatory roles of P-selectin. J. Clin. Invest. 92:559-570.

38. Shikura, S., S. Naito, H. Chaotien, K. Shichijo, Y. Kawase, M. Ito, I. Sekine, and K. Imamura. 1989. The role of the sympathetic nervous system in cysteamine-induced gastric lesions in rats. Scand. J. Gastroenterol. 162:206-209.

39. Shichijo, K., M. Ito, and I. Sekine. 1991. The mechanism of low susceptibility to stress in gastric lesions of spontaneously hypertensive rats. Life Sci. 49:2023-2029.

40. Wennberg, E., H. Hagberg, and H. Haljamae. 1984. The response of the awake spontaneously hypertensive rat (SHR) to acute blood loss. Acta Anesth. Scand. 28:457-461.

41. Barroso-Aranda, J., and G. W. Schmid-Schönbein. 1989. Transformation of neutrophils as indicator of irreversibility in hemorrhargic shock. Am. J. Physiol. 257:H699-H852. 